\title{
Plasmonic modes in thin films: quo vadis?
}

\section{Antonio Politano* and Gennaro Chiarello}

Dipartimento di Fisica, University of Calabria, Cosenza, Italy

\section{Edited by:}

Muhammad Rizwan Saleem,

University of Eastern Finland, Finland

Reviewed by:

Xiaofeng Li, Soochow University, China

Yuehui Lu, Chinese Academy of Sciences, China

*Correspondence:

Antonio Politano, Dipartimento di

Fisica, University of Calabria, Rende,

Cosenza 87036, Italy

e-mail: antonio.politano@fis.unical.it
Herein, we discuss the status and the prospect of plasmonic modes in thin films. Plasmons are collective longitudinal modes of charge fluctuation in metal samples excited by an external electric field. Surface plasmons (SPs) are waves that propagate along the surface of a conductor with applications in magneto-optic data storage, optics, microscopy, and catalysis. In thin films, the electronic response is influenced by electron quantum confinement. Confined electrons modify the dynamical screening processes at the film/substrate interface by introducing novel properties with potential applications and, moreover, they affect both the dispersion relation of SP frequency and the damping processes of the SP. Recent calculations indicate the emergence of acoustic surface plasmons (ASPs) in Ag thin films exhibiting quantum well states and in graphene films. The slope of the dispersion of ASP decreases with film thickness. We also discuss open issues in research on plasmonic modes in graphene/metal interfaces.

Keywords: thin films, plasmons, plasmonics, silver, gold, graphene, magnetoplasmonics
Plasmons in low-dimensional systems never cease to amaze with new astonishing findings, although it has quite a long history, started with the discovery of surface plasmons (SPs) in thin films by Ritchie (1957).

Recently, novel modes, such as sheet (Langer et al., 2011; Politano et al., 2012a), Dirac (Fei et al., 2011; Stauber, 2014), and acoustic surface plasmons (ASPs) (Politano et al., 2011; Yuan et al., 2011) and, moreover, plasmarons (Krstajic and Peeters, 2013), have been observed in low-dimensional systems. Such excitations are supported by the two-dimensional electron gas (2DEG). The great interest toward plasmons arises from the exceptional range of the possible applications of plasmonics.

To date, plasmonic devices based on noble metals (Ag and $\mathrm{Au}$ ) are widely diffused (Nyga et al., 2008; Pala et al., 2009). Nevertheless, current research is oriented toward the realization of graphene-based plasmonic devices. In fact, plasmons in graphene offer promising prospect of applications covering a wide frequency range, going from terahertz up to the visible (Vicarelli et al., 2012; García de Abajo, 2014).

Nanoscale thin films are an ideal playground for manipulating plasmon properties by peculiar phenomena occurring in thin films, such as quantum size effects (Hamawi et al., 1991; Wei and Chou, 2002) and quantum electron confinement (Ogando et al., 2005; Politano and Chiarello, 2010). Film morphology may originate plasmon confinement within disordered grains (Moresco et al., 1999) or periodic nanodomes (Politano et al., 2013a). Herein, the open challenges regarding plasmons modes in thin films will be presented to the reader, with a particular attention for the cases with higher prospect for plasmonic applications, i.e., noble metal ( $\mathrm{Ag}$ and $\mathrm{Au}$ ) and graphene films.

As a general rule, the electromagnetic fields of both sides forming an interface interact in such a way that the SP splits into two plasmonic excitations in which electron may oscillate in phase or not. For a Drude thin slab in vacuum of thickness $a$ (Pitarke et al.,
2007), the dispersion relations of these modes can be obtained by applying appropriate boundary conditions and solving Maxwell's equations (Raether, 1980):

$$
\omega=\frac{\omega_{p}}{\sqrt{2}}\left(1 \pm e^{-\mathrm{qa}}\right)^{1 / 2}
$$

The high energy plasmon in the Figure 1A has anti-symmetric field distribution, whereas the low-energy one has symmetric field distribution.

At short wavelengths ( $\mathrm{qa} \gg 1$ ), the surface waves become decoupled and each surface sustains independent oscillations at the reduced frequency $\omega_{s}=\omega_{p} / \sqrt{ } 2$ characteristic of a semi-infinite electron gas with a single plane boundary. At long wavelengths $(\mathrm{qa} \ll 1)$, there are normal oscillations at $\omega_{p}$ and tangential $2 \mathrm{D}$ oscillations at:

$$
\omega_{2 \mathrm{D}}=(2 \pi \mathrm{naq})^{1 / 2}
$$

which were later discussed by Stern (1967) and observed in artificially structured semiconductors (Allen et al., 1977) and, more recently, in a metallic surface-state band on a silicon surface (Nagao et al., 2001a,b).

The plasmon dispersion in Eq. 1 is modified by the interaction with phonons. Plasmon-phonon coupling is a striking manifestation of the breakdown of the Born-Oppenheimer approximation (Jablan et al., 2011), with consequences on transport (Tediosi et al., 2007) properties. The plasmon-phonon coupling phenomenon implies the hybridization of the plasmon modes of the 2DEG with the optical phonon modes, giving rise to the coupled plasmon-phonon modes (shown in Figure 1B for the sample case of graphene/ $\mathrm{SiO}_{2}$ ).

Concerning interfaces, different authors have invoked the existence of interface plasmons (Layet et al., 1986). Ahlqvist et al. (1982) have studied the electrodynamics of the interface between two 


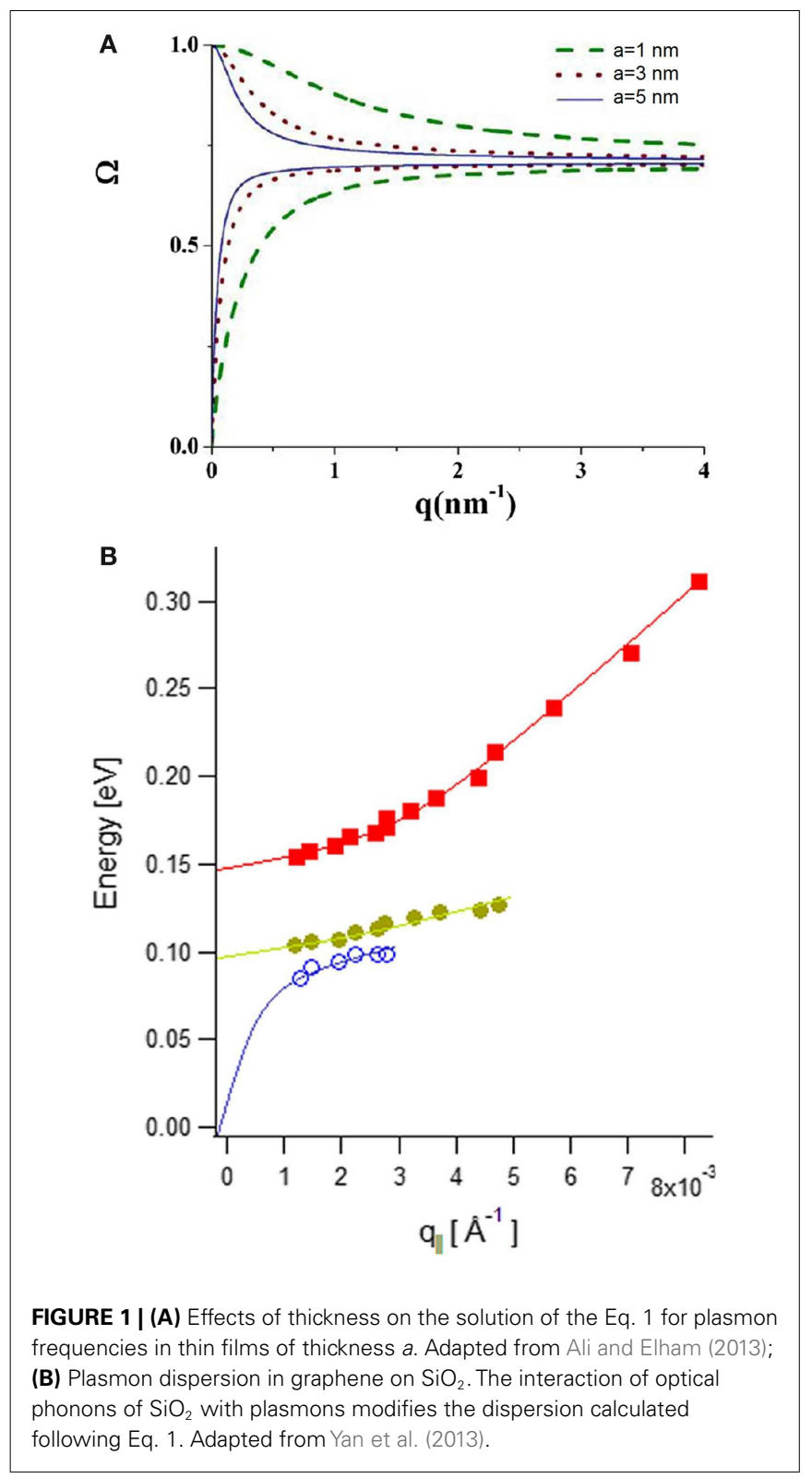

semi-infinite electron gases, finding that the interface plasmon is characterized by

$$
\omega_{i}^{2}=\left(\omega_{1}^{2}+\omega_{2}^{2}\right) / 2
$$

where $\omega_{i}, \omega_{1}$, and $\omega_{2}$ are the frequencies of the interface plasmon and of the two semi-infinite electron gases, respectively. Jewsbury and Summerside (1980) have suggested that an "interface plasmon" is not a pure mode but arises from the electronic band structure at the interface.

However, the traditional theoretical approach used to describe plasmons in thin films, based on Eqs 1 and 2 and on interface plasmons (Eq. 3) is inadequate to describe the extraordinary complexity of plasmon modes at interfaces. Thus, the overall encouraging viewpoint for plasmonic applications is also accompanied by the possibility to carry out many other fascinating fundamental studies.

As an example, the strain resulting from the lattice mismatch between adlayer and substrate (Schell-Sorokin and Tromp, 1990; Sander et al., 1998) may further affect plasmonic excitations. Additional collective electronic modes may be induced by strain, as found by Pellegrino et al. (2010) for the case of graphene. However, experimental studies are still lacking due to the difficulties in following strain effects on plasmonic excitations.

Moreover, the influence of electron quantum confinement (presence of quantum well states, QWS) on the SP is still not clearly established. Theoreticians (Yuan and Gao, 2008) and experimentalists (Yu et al., 2005; Politano et al., 2009) have put in evidence the influence of QWS on the plasmon lifetime in films. Due to the opening of the decay channel of the SP into electron-hole pairs via interband transitions involving QWS, the line-width of the SP assumes an unusual dispersion relation as a function of the momentum transfer, as compared with the case of bulk samples. The effects of QWS on plasmon dispersion have been studied only for a few systems. In Politano et al. (2008) and Politano and Chiarello (2009), it has been shown that the screening properties are influenced by the presence of the modified electron distribution in the presence of QWS. However, rigorous and satisfactory theoretical description is still missing.

The presence of QWS and the subsequent enhanced SP density of states around the Fermi level in thin films may also increase the cross section for the excitation of intrinsically free-electron plasmons, such as the multipole surface plasmon (MP) (Liebsch, 1998). The nature of MP has been understood for alkali metals (Tsuei et al., 1990, 1991; Sprunger et al., 1992; Zielasek et al., 2006), alkaline-earth metals (Sprunger et al., 1992), and aluminum (Chiarello et al., 2000). Unfortunately, contradictory results are reported for the most popular plasmonic systems (Ag and $\mathrm{Au}$ ). Calculations based on a s-d polarization model by Liebsch (1998) predicted the existence of the Ag and Au MP near $\omega_{m}=0.8 \cdot \omega_{p}=7.2 \mathrm{eV}\left(\omega_{p}=9.0 \mathrm{eV}\right.$ is the s-electron bulk plasmon energy for both $\mathrm{Ag}$ and $\mathrm{Au}$ ) as the density profile at the surface has predominantly s-electron character. Experiments on bulk Ag have not found this mode (Moresco et al., 1996; Barman et al., 2004a,b). In contrast, the Ag MP has been recently measured in $\mathrm{Ag}$ films on $\mathrm{Ni}(111)$. However, such excitation is revealed only in experimental conditions enhancing the surface sensitivity, i.e., at low impinging energies and grazing incidence (Politano et al., 2013b,d), in agreement with Liebsch's prediction (Liebsch, 1998). Therefore, electron quantum confinement in Ag 5 sp-derived QWS (Miller et al., 1994) enhances the cross section for Ag MP excitation in thin films compared with semi-infinite media (bulk samples).

Another open issue is related to the possible existence of acoustic plasmon modes in thin films. Unfortunately, to date no experimental works exist on this topic, while from the theoretical side Silkin et al. (2011) have shown that ASP emerge in the electronic response of thin Ag films. The presence of Ag QWS in 

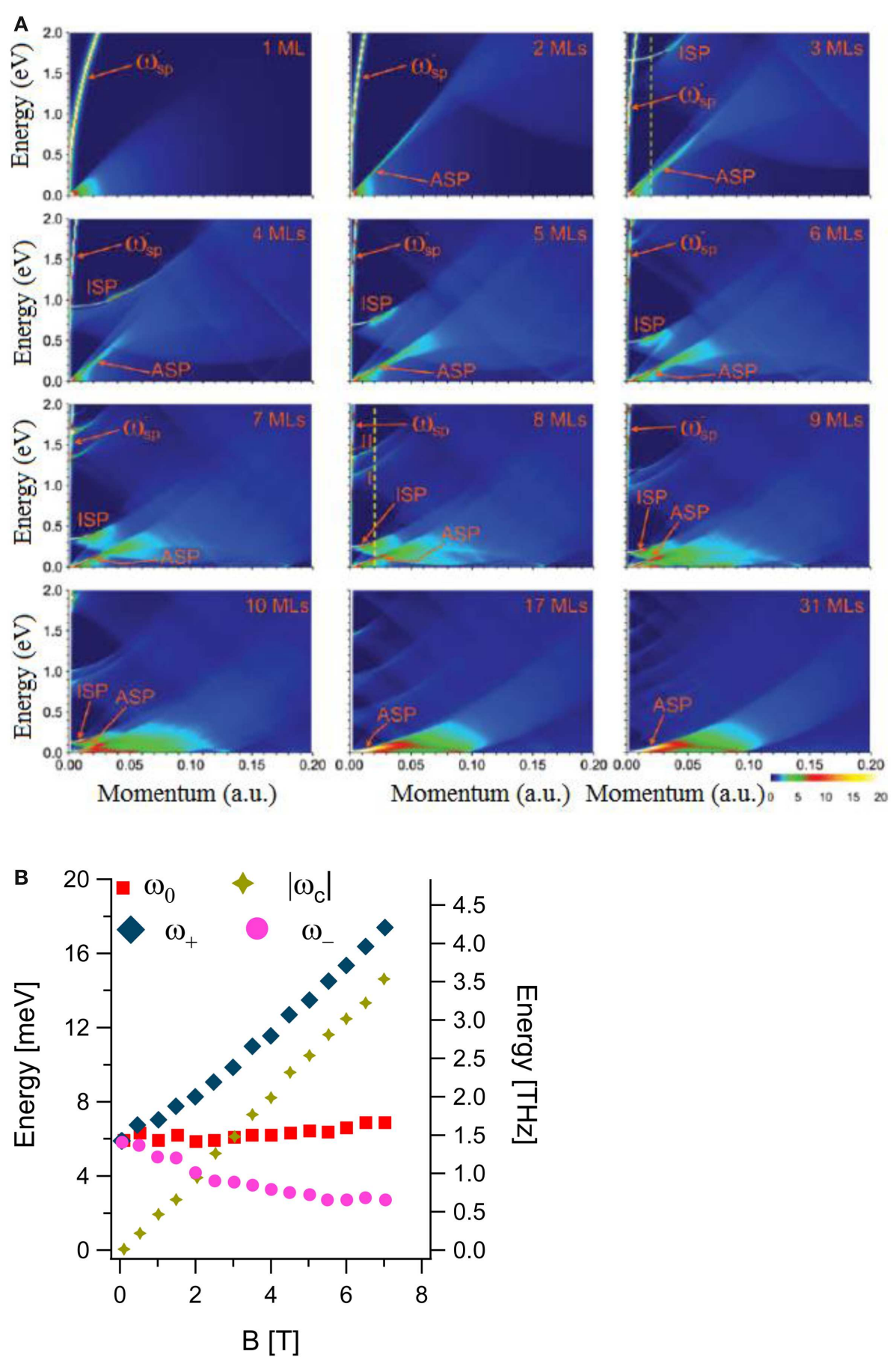

FIGURE 2 | (A) Normalized surface loss function $\operatorname{Im}[g(q, \omega)] / q \omega$ for $\mathrm{Ag}(111)$ films with thickness ranging from 1 to 31 monolayers (ML) evaluated by using realistic effective masses in energy band

dispersions. Note the strongly dispersing mode corresponding to a conventional ${ }^{\omega_{\mathrm{SP}}^{-}}$mode of a thin film. Peaks denoted with "ISP" are originated from the interband transition between the energy-split quantum states. Adapted from Silkin et al. (2011). (B) Dependence of the plasmon energy $\omega_{0}$, the cyclotron resonance energy $\omega_{C}$ and the magnetoplasmon energies $\omega_{ \pm}$on the magnetic field B. Adapted from Crassee et al. (2012). ultrathin films induces the appearance of ASP, whose dispersion is determined by the QWS band. The slope of the dispersion relation decreases with film thickness.
The surface response function (Figure 2A) for film thickness higher than three layers shows an additional feature at about $2 \mathrm{eV}$, which correspond to interband transitions between energy-split 
$\mathrm{SS}^{+}$and $\mathrm{SS}^{-}$electronic states (interband SP, ISP). In contrast with ASP, the ISP energy has finite value at $q=0$. Moreover, the ISP energy decreases with increasing thickness and it merges with the ASP at higher thickness.

Acoustic surface plasmon owes its existence to the spatial coexistence of a $2 \mathrm{DEG}$ with a $3 \mathrm{D}$ electron gas. It has been also predicted to exist at the K/Be interface (Echeverry et al., 2010; Silkin et al., 2010a,b). The screening by the underlying metal substrate change the square-root-like dispersion of the $2 \mathrm{D}$ plasmon into linear.

Concerning graphene films, the most puzzling open issues are related to plasmonic modes in graphene/metal interfaces. Due to the difficulty in the theoretical description of the screening by the underlying metal substrate, accurate theoretical models for plasmons in graphene/metal interfaces are still missing. The out-of-plane charge transfer between graphene and the metal is determined by the difference between the work function of graphene and the metal surface and, in addition, by the metalgraphene chemical interaction that creates an interface dipole lowering the metal work function. The induced electrostatic potential decays weakly with the distance from the metal contact as $V(x) \approx x^{-1 / 2}$ and $\approx x^{-1}$ for undoped and doped graphene, respectively (Khomyakov et al., 2010). Instead, current models overestimate the screening by the metal substrate. Likely, the experimental study of plasmons in graphene deposited on jellium surfaces $(\mathrm{Al})$ could help theoreticians to improve our understanding of screening processes at graphene/metals. Unfortunately, such experimental study is complicated by the difficult preparation of graphene on jellium surfaces.

Low-energy intraband plasmon in graphene is currently well understood (Shin et al., 2011; Stauber and Gómez-Santos, 2012b; Stauber, 2014). In contrast, theoretical models hitherto fail to describe the nature of a non-linear mode observed at $\sim 0.5 \mathrm{eV}$ (Politano and Chiarello, 2014) and, moreover, the quadratic dispersion of interband plasmon (Generalov and Dedkov, 2012; Politano et al., 2012b) in graphene/metal interfaces. The dispersion of the interband plasmon is instead linear in both freestanding graphene (Kramberger et al., 2008) and Cs-decoupled graphene/Ni(111) (Cupolillo et al., 2013b; Ligato et al., 2013). The observation of the change of the interband plasmon from linear to quadratic as a function of the number of graphene layers on silicon carbide (Lu et al., 2009) may in principle afford important information for shedding light on the still confusing state-of-theart of plasmon modes in epitaxial graphene. However, theoretical models describing the increasing wealth of experimental results on interband plasmons are yet missing.

Moreover, experimental studies on plasmons in bilayer graphene grown on metals would be essential to verify and improve current theoretical models for both plasmon dispersion(Wang and Chakraborty, 2007; Sensarma et al., 2010; Stauber and GómezSantos, 2012a; Roldán and Brey, 2013) and plasmaron formation (Van-Nham and Holger, 2012; Krstajic and Peeters, 2013).

Finally, another intriguing topic is magnetoplasmonics, which recently is attracting huge interest for its potential applications in technology (Belotelov et al., 2011; Bonanni et al., 2011). The $2 \mathrm{D}$ magnetoplasmons are collective excitations between Landau levels (Lozovik and Sokolik, 2012). They can be observed through infrared optical absorption and inelastic light scattering (Kallin and Halperin, 1984; Oji and MacDonald, 1986; Cinà et al., 1999; Eriksson et al., 1999; Bychkov and Martinez, 2002; Li and Zhai, 2011). In layered and doped graphene structures, the instability and unusual dispersion of magnetoplasmon modes have been studied in recent years, within different approaches (Tahir and Sabeeh, 2007; Berman et al., 2008, 2009; Bychkov and Martinez, 2008a,b; Fischer et al., 2009, 2010; Roldán et al., 2009; Tahir et al., 2011; Wu et al., 2011; Bisti and Kirova, 2012; Ferreira et al., 2012; Lozovik and Sokolik, 2012; Wang et al., 2012; Yan et al., 2012; Chamanara et al., 2013a,b; Petkovic et al., 2013). Magnetoplasmons have been observed in graphene epitaxially grown on SiC (Crassee et al., 2012). The Drude absorption is transformed into a strong terahertz plasmonic peak due to nanoscale inhomogeneities, such as substrate terraces and wrinkles. Plasmonic excitations also modify the magneto-optical response and, in particular, the Faraday rotation (Crassee et al., 2012). This makes graphene a unique playground for plasmon-controlled magnetooptical phenomena thanks to a cyclotron mass, which is two orders of magnitude smaller than in conventional plasmonic materials, such as noble metals.

The field-induced splitting of the plasmon peak resembles strikingly the appearance of collective resonances previously observed in other systems (Allen et al., 1983; Glattli et al., 1985; Mast et al., 1985; Kukushkin et al., 2003). The upper and lower branches are attributed to the so-called bulk and edge magnetoplasmons, respectively, with the frequencies

$$
\omega_{ \pm}=\sqrt{\frac{\omega_{c}^{2}}{4}+\omega_{0}^{2}} \pm \frac{\left|\omega_{c}\right|}{2}
$$

where $\omega_{0}$ is the plasmon frequency at 0 field, $\omega_{c}= \pm e B / m c$ is the cyclotron frequency, defined as positive for electrons and negative for holes, $m$ is the cyclotron mass, and $c$ the speed of light. At high fields $\left(\left|\omega_{c}\right| \gg \omega_{0}\right)$, the upper branch becomes essentially the usual cyclotron resonance with a linear dependence on magnetic field, while the lower branch represents a collective mode confined to the edges (Fetter, 1985) with the energy inversely proportional to the field (Figure 2B).

In conclusion, issues discussed herein provide the grounds for theoretical studies aimed at characterizing in more details how growth mode, quantum size effects, and the electron quantum confinement within the adlayer influence the dispersion and the lifetime of collective excitations in nanoscale thin films.

The comprehension of plasmonic excitations in thin films (Chiarello et al., 1997a,b), especially of noble metals (Politano and Chiarello, 2009; Politano, 2012a,b, 2013) and graphene (Politano et al., 2011, 2012a,b, 2013a,c; Cupolillo et al., 2012, 2013a,b; Politano and Chiarello, 2013a,b, 2014), could keep active researchers for a long time.

\section{REFERENCES}

Ahlqvist, P., Monreal, R., Flores, F., and Garcia-Moliner, F. (1982). Interface plasmons at the boundary of two semi-infinite electron gases. Phys. Scripta 26, 35. doi:10.1088/0031-8949/26/1/006 
Ali, B., and Elham, A. (2013). Effect of shell thickness on propagation of surface hybrid modes in metallic cylindrical nanoshells. Phys. Scripta 88, 035707. doi:10.1088/0031-8949/88/03/035707

Allen, S. J., Störmer, H. L., and Hwang, J. C. M. (1983). Dimensional resonance of the two-dimensional electron gas in selectively doped GaAs/AlGaAs heterostructures. Phys. Rev. B 28, 4875-4877. doi:10.1103/PhysRevB.28.4875

Allen, S. J., Tsui, D. C., and Logan, R. A. (1977). Observation of the twodimensional plasmon in silicon inversion layers. Phys. Rev. Lett. 38, 980 doi:10.1103/PhysRevLett.38.980

Barman, S. R., Biswas, C., and Horn, K. (2004a). Collective excitations on silver surfaces studied by photoyield. Surf. Sci. 566-568, 538-543. doi:10.1016/j.susc. 2004.06.059

Barman, S. R., Biswas, C., and Horn, K. (2004b). Electronic excitations on silver surfaces. Phys. Rev. B 69, 454131-454139. doi:10.1103/PhysRevB.69.045413

Belotelov, V. I., Akimov, I. A., Pohlm, M., Kotov, V. A., Kastures, S., Vengurlekar, A. S., et al. (2011). Enhanced magneto-optical effects in magnetoplasmonic crystals. Nat. Nanotechnol. 6, 370-376. doi:10.1038/nnano.2011.54

Berman, O. L., Gumbs, G., and Echenique, P. M. (2009). Quasiparticles for a quantum dot array in graphene and the associated magnetoplasmons. Phys. Rev. B 79, 075418. doi:10.1103/PhysRevB.79.075418

Berman, O. L., Gumbs, G., and Lozovik, Y. E. (2008). Magnetoplasmons in layered graphene structures. Phys. Rev. B 78, 085401. doi:10.1103/PhysRevB.78.085401

Bisti, V. E., and Kirova, N. N. (2012). Cyclotron excitations in pure bilayer graphene: electron-hole asymmetry and coulomb interaction. Physica B Condens. Matter 407, 1923-1926. doi:10.1016/j.physb.2012.01.065

Bonanni, V., Bonetti, S., Pakizeh, T., Pirzadeh, Z., Chen, J., Nogués, J., et al. (2011). Designer magnetoplasmonics with nickel nanoferromagnets. Nano Lett. 11, 5333-5338. doi:10.1021/nl2028443

Bychkov, Y. A., and Martinez, G. (2002). Magnetoplasmons and band nonparabolicity in two-dimensional electron gas. Phys. Rev. B 66, 193312. doi:10.1103/ PhysRevB.66.193312

Bychkov, Y. A., and Martinez, G. (2008a). Magnetoplasmon excitations in graphene. Physica ELow Dimens. Syst. Nanostruct. 40, 1410-1411. doi:10.1016/j.physe.2007. 09.026

Bychkov, Y. A., and Martinez, G. (2008b). Magnetoplasmon excitations in graphene for filling factors $v \leq 6$. Phys. Rev. B 77, 125417. doi:10.1103/PhysRevB.77.125417

Chamanara, N., Sounas, D., and Caloz, C. (2013a). Non-reciprocal magnetoplasmon graphene coupler. Opt. Express 21, 11248-11256. doi:10.1364/OE.21.011248

Chamanara, N., Sounas, D., Szkopek, T., and Caloz, C. (2013b). Terahertz magnetoplasmon energy concentration and splitting in graphene $\mathrm{PN}$ junctions. Opt. Express 21, 25356-25363. doi:10.1364/OE.21.025356

Chiarello, G., Cupolillo, A., Amoddeo, A., Caputi, L. S., Papagno, L., and Colavita, E. (1997a). Collective excitations of two layers of K on Ni(111). Phys. Rev. B 55 1376-1379. doi:10.1103/PhysRevB.55.1376

Chiarello, G., Cupolillo, A., Caputi, L. S., Papagno, L., and Colavita, E. (1997b). Collective and single-particle excitations in thin layers of $\mathrm{K}$ on $\mathrm{Ni}(111)$. Surf. Sci. 377, 365-370. doi:10.1016/S0039-6028(96)01419-7

Chiarello, G., Formoso, V., Santaniello, A., Colavita, E., and Papagno, L. (2000). Surface-plasmon dispersion and multipole surface plasmons in $\mathrm{Al}(111)$. Phys Rev. B 62, 12676-12679. doi:10.1103/PhysRevB.62.12676

Cinà, S., Whittaker, D. M., Arnone, D. D., Burke, T., Hughes, H. P., Leadbeater, M., et al. (1999). Magnetoplasmons in a tunable periodically modulated magnetic field. Phys. Rev. Lett. 83, 4425-4428. doi:10.1103/PhysRevLett.83.4425

Crassee, I., Orlita, M., Potemski, M., Walter, A. L., Ostler, M., Seyller, T., et al. (2012). Intrinsic terahertz plasmons and magnetoplasmons in large scale monolayer graphene. Nano Lett. 12, 2470-2474. doi:10.1021/nl300572y

Cupolillo, A., Ligato, N., and Caputi, L. (2013a). Low energy two-dimensional plasmon in epitaxial graphene on $\mathrm{Ni}$ (111). Surf. Sci. 608, 88-91. doi:10.1016/j.susc. 2012.09.018

Cupolillo, A., Ligato, N., and Caputi, L. S. (2013b). Plasmon dispersion in quasifreestanding graphene on Ni(111). Appl. Phys. Lett. 102, 111609. doi:10.1063/1. 4798331

Cupolillo, A., Ligato, N., and Caputi, L. S. (2012). Two-dimensional character of the interface- $\pi$ plasmon in epitaxial graphene on $\mathrm{Ni}(111)$. Carbon N. Y. 50 , 2588-2591. doi:10.1016/j.carbon.2012.02.017

Echeverry, J. P., Chulkov, E. V., and Silkin, V. M. (2010). Collective electronic excitations in a potassium-covered BE surface. Phys. Status Solidi C 7, 2640-2643. doi:10.1002/pssc. 200983842
Eriksson, M. A., Pinczuk, A., Dennis, B. S., Simon, S. H., Pfeiffer, L. N., and West, K. W. (1999). Collective excitations in the dilute 2D electron system. Phys. Rev. Lett. 82, 2163-2166. doi:10.1103/PhysRevLett.82.2163

Fei, Z., Andreev, G. O., Bao, W., Zhang, L. M., Mcleod, S., Wang, C., et al. (2011). Infrared nanoscopy of dirac plasmons at the graphene-SiO ${ }_{2}$ interface. Nano Lett. 11, 4701-4705. doi:10.1021/nl202362d

Ferreira, A., Peres, N. M. R., and Castro Neto, A. H. (2012). Confined magnetooptical waves in graphene. Phys. Rev. B 85, 205426. doi:10.1103/PhysRevB.85. 205426

Fetter, A. L. (1985). Edge magnetoplasmons in a bounded two-dimensional electron fluid. Phys. Rev. B 32, 7676-7684. doi:10.1103/PhysRevB.32.7676

Fischer, A. M., Dzyubenko, A. B., and Römer, R. A. (2009). Localized collective excitations in doped graphene in strong magnetic fields. Phys. Rev. B 80, 165410. doi:10.1103/PhysRevB.80.165410

Fischer, A. M., Römer, R. A., and Dzyubenko, A. B. (2010). Symmetry content and spectral properties of charged collective excitations for graphene in strong magnetic fields. Europhys. Lett. 92, 37003. doi:10.1209/0295-5075/92/37003

García de Abajo, F. J. (2014). Graphene plasmonics: challenges and opportunities. ACS Photonics 1, 135-152. doi:10.1021/ph400147y

Generalov, A. V., and Dedkov, Y. S. (2012). EELS study of the epitaxial graphene/Ni(111) and graphene/Au/Ni(111) systems. Carbon N. Y. 50, 183-191. doi:10.1016/j.carbon.2011.08.018

Glattli, D. C., Andrei, E. Y., Deville, G., Poitrenaud, J., and Williams, F. I. B. (1985). Dynamical hall effect in a two-dimensional classical plasma. Phys. Rev. Lett. 54, 1710-1713. doi:10.1103/PhysRevLett.54.1710

Hamawi, A., Lindgren, S. A., and Walldén, L. (1991). Quantum size effects in thin metal overlayers. Phys. Scripta T39, 339-345. doi:10.1088/0031-8949/1991/ T39/053

Jablan, M., Soljacic, M., and Buljan, H. (2011). Unconventional plasmon-phonon coupling in graphene. Phys. Rev. B 83, 161409. doi:10.1103/PhysRevB.83.161409

Jewsbury, P., and Summerside, P. (1980). The nature of interface plasmon modes at bimetallic junctions. J. Phys. F Met. Phys. 10, 645. doi:10.1088/0305-4608/10/ $4 / 015$

Kallin, C., and Halperin, B. I. (1984). Excitations from a filled Landau level in the twodimensional electron gas. Phys. Rev. B 30, 5655-5668. doi:10.1103/PhysRevB.30. 5655

Khomyakov, P. A., Starikov, A. A., Brocks, G., and Kelly, P. J. (2010). Nonlinear screening of charges induced in graphene by metal contacts. Phys. Rev. B 82, 115437. doi:10.1103/PhysRevB.82.115437

Kramberger, C., Hambach, R., Giorgetti, C., Rümmeli, M. H., Knupfer, M., Fink, J., et al. (2008). Linear plasmon dispersion in single-wall carbon nanotubes and the collective excitation spectrum of graphene. Phys. Rev. Lett. 100, 196803. doi:10.1103/PhysRevLett.100.196803

Krstajic, P. M., and Peeters, F. M. (2013). Energy-momentum dispersion relation of plasmarons in bilayer graphene. Phys. Rev. B 88, 165420. doi:10.1103/PhysRevB. 88.165420

Kukushkin, I. V., Smet, J. H., Mikhailov, S. A., Kulakovskii, D. V., Von Klitzing, K., and Wegscheider, W. (2003). Observation of retardation effects in the spectrum of two-dimensional plasmons. Phys. Rev. Lett. 90, 156801. doi:10.1103/ PhysRevLett.90.156801

Langer, T., Förster, D. F., Busse, C., Michely, T., Pfnür, H., and Tegenkamp, C. (2011). Sheet plasmons in modulated graphene on $\operatorname{Ir}(111)$. New J. Phys. 13, 053006 doi:10.1088/1367-2630/13/5/053006

Layet, J. M., Contini, R., Derrien, J., and Lüth, H. (1986). Coupled interface plasmons of the Ag-Si(111) system as investigated with high-resolution electron energy-loss spectroscopy. Surf. Sci. 168, 142-148. doi:10.1016/0039-6028(86) 90844-7

Li, C., and Zhai, F. (2011). Anisotropic magnetoplasmon spectrum of twodimensional electron gas systems with the Rashba and Dresselhaus spin-orbit interactions. J. Appl. Phys. 109, 093306. doi:10.1063/1.3583651

Liebsch, A. (1998). Prediction of a Ag multipole surface plasmon. Phys. Rev. B 57, 3803-3806. doi:10.1103/PhysRevB.57.3803

Ligato, N., Cupolillo, A., and Caputi, L. S. (2013). Study of the intercalation of graphene on $\mathrm{Ni}(111)$ with Cs atoms: towards the quasi-free graphene. Thin Solid Films 543, 59-62. doi:10.1016/j.tsf.2013.02.121

Lozovik, Y. E., and Sokolik, A. A. (2012). Influence of Landau level mixing on the properties of elementary excitations in graphene in strong magnetic field. Nanoscale Res. Lett. 7, 1-19. doi:10.1186/1556-276X-7-134 
Lu, J., Loh, K. P., Huang, H., Chen, W., and Wee, A. T. S. (2009). Plasmon dispersion on epitaxial graphene studied using high-resolution electron energy-loss spectroscopy. Phys. Rev. B 80, 113410. doi:10.1088/0953-8984/23/1/012001

Mast, D. B., Dahm, A. J., and Fetter, A. L. (1985). Observation of bulk and edge magnetoplasmons in a two-dimensional electron fluid. Phys. Rev. Lett. 54, 1706-1709. doi:10.1103/PhysRevLett.54.1706

Miller, T., Samsavar, A., and Chiang, T. C. (1994). Photoexcitation of resonances in Ag films on Ni(111). Phys. Rev. B 50, 17686. doi:10.1103/PhysRevB.50.17686

Moresco, F., Rocca, M., Hildebrandt, T., and Henzler, M. (1999). Plasmon confinement in ultrathin continuous Ag films. Phys. Rev. Lett. 83, 2238-2241. doi:10.1103/PhysRevLett.83.2238

Moresco, F., Rocca, M., Zielasek, V., Hildebrandt, T., and Henzler, M. (1996). Evidence for the presence of the multipole plasmon mode on Ag surfaces. Phys. Rev. B 54, 14333-14336. doi:10.1103/PhysRevB.54.R14333

Nagao, T., Hildebrandt, T., Henzler, M., and Hasegawa, S. (2001a). Dispersion and damping of a two-dimensional plasmon in a metallic surface-state band. Phys. Rev. Lett. 86, 5747-5750. doi:10.1103/PhysRevLett.86.5747

Nagao, T., Hildebrandt, T., Henzler, M., and Hasegawa, S. (2001b). Two-dimensional plasmon in a surface-state band. Surf. Sci. 493, 680-686. doi:10.1016/S00396028(01)01282-1

Nyga, P., Drachev, V. P., Thoreson, M. D., and Shalaev, V. M. (2008). Mid-IR plasmonics and photomodification with Ag films. Appl. Phys. B 93, 59-68. doi:10.1007/s00340-008-3145-9

Ogando, E., Zabala, N., Chulkov, E. V., and Puska, M. J. (2005). Self-consistent study of electron confinement to metallic thin films on solid surfaces. Phys. Rev. B 71 205401. doi:10.1103/PhysRevB.71.205401

Oji, H. C. A., and MacDonald, A. H. (1986). Magnetoplasma modes of the two-dimensional electron gas at nonintegral filling factors. Phys. Rev. B 33, 3810-3818. doi:10.1103/PhysRevB.33.3810

Pala, R. A., White, J., Barnard, E., Liu, J., and Brongersma, M. L. (2009). Design of plasmonic thin-film solar cells with broadband absorption enhancements. Adv. Mater. Weinheim 21, 3504-3509. doi:10.1002/adma.200900331

Pellegrino, F. M. D., Angilella, G. G. N., and Pucci, R. (2010). Dynamical polarization of graphene under strain. Phys. Rev. B 82, 115434. doi:10.1103/PhysRevB 82.115434

Petkovic, I., Williams, F. I. B., Bennaceur, K., Portier, F., Roche, P., and Glattli, D. C. (2013). Carrier drift velocity and edge magnetoplasmons in graphene. Phys. Rev. Lett. 110, 016801. doi:10.1103/PhysRevLett.110.016801

Pitarke, J. M., Silkin, V. M., Chulkov, E. V., and Echenique, P. M. (2007). Theory of surface plasmons and surface-plasmon polaritons. Rep. Prog. Phys. 70, 1-87. doi:10.1088/0034-4885/70/1/R01

Politano, A. (2012a). Influence of structural and electronic properties on the collective excitations of $\mathrm{Ag} / \mathrm{Cu}(111)$. Plasmonics 7, 131-136. doi:10.1007/s11468-0119285-5

Politano, A. (2012b). Interplay of structural and temperature effects on plasmonic excitations at noble-metal interfaces. Philos. Mag. 92, 768-778. doi:10.1080/ 14786435.2011.634846

Politano, A. (2013). Low-energy collective electronic mode at a noble metal interface. Plasmonics 8, 357-360. doi:10.1007/s11468-012-9397-6

Politano, A., Agostino, R. G., Colavita, E., Formoso, V., and Chiarello, G. (2008). Purely quadratic dispersion of surface plasmon in $\mathrm{Ag} / \mathrm{Ni}(111)$ : the influence of electron confinement. Phys. Status Solidi Rapid Res. Lett. 2, 86-88. doi:10.1002/pssr.200701307

Politano, A., Campi, D., Formoso, V., and Chiarello, G. (2013a). Evidence of confinement of the $\pi$ plasmon in periodically rippled graphene on $\mathrm{Ru}(0001)$. Phys Chem. Chem. Phys. 15, 11356-11361. doi:10.1039/c3cp51954f

Politano, A., Formoso, V., and Chiarello, G. (2013b). Collective electronic excitations in thin Ag films on Ni(111). Plasmonics 8, 1683-1690. doi:10.1007/s1146811013-19587-x

Politano, A., Formoso, V., and Chiarello, G. (2013c). Evidence of composite plasmonphonon modes in the electronic response of epitaxial graphene. J. Phys. Condens. Matter 25, 345303. doi:10.1088/0953-8984/25/34/345303

Politano, A., Formoso, V., and Chiarello, G. (2013d). Interplay between singleparticle and plasmonic excitations in the electronic response of thin Ag films. J. Phys. Condens. Matter 25, 305001. doi:10.1088/0953-8984/25/30/305001

Politano, A., and Chiarello, G. (2009). Collective electronic excitations in systems exhibiting quantum well states. Surf. Rev. Lett. 16, 171-190. doi:10.1142/ S0218625X09012482
Politano, A., and Chiarello, G. (2010). Enhancement of hydrolysis in alkali ultrathin layers on metal substrates in the presence of electron confinement. Chem. Phys. Lett. 494, 84-87. doi:10.1016/j.cplett.2010.05.089

Politano, A., and Chiarello, G. (2013a). Quenching of plasmons modes in airexposed graphene-Ru contacts for plasmonic devices. Appl. Phys. Lett. 102, 201608. doi:10.1039/c3nr02027d

Politano, A., and Chiarello, G. (2013b). Unravelling suitable graphene-metal contacts for graphene-based plasmonic devices. Nanoscale 5, 8215-8220. doi:10. 1039/c3nr02027d

Politano, A., and Chiarello, G. (2014). Emergence of a nonlinear plasmon in the electronic response of doped graphene. Carbon N. Y. 71, 176-180. doi:10.1016/ j.carbon.2014.01.026

Politano, A., Formoso, V., and Chiarello, G. (2009). Damping of the surface plasmon in clean and K-modified Ag thin films. J. Electron Spectros. Relat. Phenomena 173, 12-17. doi:10.1016/j.elspec.2009.03.003

Politano, A., Marino, A. R., and Chiarello, G. (2012a). Effects of a humid environment on the sheet plasmon resonance in epitaxial graphene. Phys. Rev. B 86, 085420. doi:10.1103/PhysRevB.86.085420

Politano, A., Marino, A. R., Formoso, V., Farías, D., Miranda, R., and Chiarello, G. (2012b). Quadratic dispersion and damping processes of $\pi$ plasmon in monolayer graphene on $\mathrm{Pt}(111)$. Plasmonics 7, 369-376. doi:10.1007/s11468-0119317- 1

Politano, A., Marino, A. R., Formoso, V., Farías, D., Miranda, R., and Chiarello, G. (2011). Evidence for acoustic-like plasmons on epitaxial graphene on $\operatorname{Pt}(111)$. Phys. Rev. B 84, 033401. doi:10.1103/PhysRevB.84.033401

Raether, H. (1980). Excitation of Plasmons and Interband Transitions by Electrons. Berlin: Springer-Verlag.

Ritchie, R. H. (1957). Plasma losses by fast electrons in thin films. Phys. Rev. B 106, 874-881. doi:10.1103/PhysRev.106.874

Roldán, R., and Brey, L. (2013). Dielectric screening and plasmons in AA-stacked bilayer graphene. Phys. Rev. B 88, 115420. doi:10.1103/PhysRevB. 88.115420

Roldán, R., Fuchs, J. N., and Goerbig, M. O. (2009). Collective modes of doped graphene and a standard two-dimensional electron gas in a strong magnetic field: linear magnetoplasmons versus magnetoexcitons. Phys. Rev. B 80, 085408. doi:10.1103/PhysRevB.80.085408

Sander, D., Schmidthals, C., Enders, A., and Kirschner, J. (1998). Stress and structure of Ni monolayers on W(110): the importance of lattice mismatch. Phys. Rev. B 57, 1406-1409. doi:10.1103/PhysRevB.57.1406

Schell-Sorokin, A. J., and Tromp, R. M. (1990). Mechanical stresses in (sub)monolayer epitaxial films. Phys. Rev. Lett. 64, 1039-1042. doi:10.1103/ PhysRevLett.64.1039

Sensarma, R., Hwang, E. H., and Das Sarma, S. (2010). Dynamic screening and low-energy collective modes in bilayer graphene. Phys. Rev. B 82, 195428. doi:10.1088/0957-4484/23/50/505204

Shin, S. Y., Hwang, C. G., Sung, S. J., Kim, N. D., Kim, H. S., and Chung, J. W. (2011). Observation of intrinsic intraband $\pi$-plasmon excitation of a single-layer graphene. Phys. Rev. B 83, 161403. doi:10.1103/PhysRevB.83.161403

Silkin, V. M., Chulkov, E. V., Echeverry, J. P., and Echenique, P. M. (2010a). Modification of response properties of the $\mathrm{Be}(0001)$ surface upon adsorption of a potassium monolayer: an Ab initio calculation. Phys. Status Solidi B 247, 1849-1857. doi: $10.1002 /$ pssb. 200983843

Silkin, V. M., Hellsing, B., Walldén, L., Echenique, P. M., and Chulkov, E. V. (2010b). Photoelectron driven acoustic surface plasmons in $\mathrm{p}(2 \times 2) \mathrm{K} / \mathrm{Be}(0001)$ : Ab initio calculations. Phys. Rev. B 81, 113406. doi:10.1103/PhysRevB.81.113406

Silkin, V. M., Nagao, T., Despoja, V., Echeverry, J. P., Eremeev, S. V., Chulkov, E. V., et al. (2011). Low-energy plasmons in quantum-well and surface states of metallic thin films. Phys. Rev. B 84, 165416. doi:10.1103/PhysRevB.84. 165416

Sprunger, P. T., Watson, G. M., and Plummer, E. W. (1992). The normal modes at the surface of Li and Mg. Surf. Sci. 269-270, 551-555. doi:10.1016/0039-6028(92) 91307-W

Stauber, T. (2014). Plasmonics in Dirac systems: from graphene to topological insulators. J. Phys. Condens. Matter 26, 123201. doi:10.1088/0953-8984/26/12/ 123201

Stauber, T., and Gómez-Santos, G. (2012a). Plasmons and near-field amplification in double-layer graphene. Phys. Rev. B 85, 075410. doi:10.1103/PhysRevB.85. 075410 
Stauber, T., and Gómez-Santos, G. (2012b). Plasmons in layered structures including graphene. New J. Phys. 14, 105018. doi:10.1088/1367-2630/14/10/105018

Stern, F. (1967). Polarizability of a two-dimensional electron gas. Phys. Rev. Lett. 18, 546-548. doi:10.1103/PhysRevLett.18.546

Tahir, M., and Sabeeh, K. (2007). Theory of Weiss oscillations in the magnetoplasmon spectrum of Dirac electrons in graphene. Phys. Rev. B 76, 195416. doi:10.1103/PhysRevB.76.195416

Tahir, M., Sabeeh, K., and Mackinnon, A. (2011). Temperature effects on the magnetoplasmon spectrum of a weakly modulated graphene monolayer. J. Phys. Condens. Matter 23, 425304. doi:10.1088/0953-8984/23/42/425304

Tediosi, R., Armitage, N. P., Giannini, E., and Van Der Marel, D. (2007). Charge carrier interaction with a purely electronic collective mode: plasmarons and the infrared response of elemental bismuth. Phys. Rev. Lett. 99, 016406. doi:10.1103/PhysRevLett.99.016406

Tsuei, K. D., Plummer, E. W., Liebsch, A., Kempa, K., and Bakshi, P. (1990). Multipole plasmon modes at a metal surface. Phys. Rev. Lett. 64, 44-47. doi:10.1103/PhysRevLett.64.44

Tsuei, K. D., Plummer, E. W., Liebsch, A., Pehlke, E., Kempa, K., and Bakshi, P. (1991). The normal modes at the surface of simple metals. Surf. Sci. 247, 302-326. doi:10.1016/0039-6028(91)90142-F

Van-Nham, P., and Holger, F. (2012). Coulomb interaction effects in graphene bilayers: electron-hole pairing and plasmaron formation. New J. Phys. 14, 075007. doi:10.1088/1367-2630/14/7/075007

Vicarelli, L., Vitiello, M., Coquillat, D., Lombardo, A., Ferrari, A., Knap, W., et al. (2012). Graphene field-effect transistors as room-temperature terahertz detectors. Nat. Mater. 11, 865-871. doi:10.1038/nmat3417

Wang, W., Apell, S. P., and Kinaret, J. M. (2012). Edge magnetoplasmons and the optical excitations in graphene disks. Phys. Rev. B 86, 125450. doi:10.1021/nl3016335

Wang, X.-F., and Chakraborty, T. (2007). Coulomb screening and collective excitations in a graphene bilayer. Phys. Rev. B 75, 041404. doi:10.1103/PhysRevB.75. 041404

Wei, C. M., and Chou, M. Y. (2002). Theory of quantum size effects in thin $\mathrm{Pb}(111)$ films. Phys. Rev. B 66, 233408. doi:10.1103/PhysRevB.66.233408

Wu, J. Y., Chen, S. C., Roslyak, O., Gumbs, G., and Lin, M. F. (2011). Plasma excitations in graphene: their spectral intensity and temperature dependence in magnetic field. ACS Nano 5, 1026-1032. doi:10.1021/nn1024847
Yan, H., Li, Z., Li, X., Zhu, W., Avouris, P., and Xia, F. (2012). Infrared spectroscopy of tunable Dirac terahertz magneto-plasmons in graphene. Nano Lett. 12, 3766-3771. doi:10.1021/nl3016335

Yan, H., Low, T., Zhu, W., Wu, Y., Freitag, M., Li, X., et al. (2013). Damping pathways of mid-infrared plasmons in graphene nanostructures. Nat. Photonics 7 , 394-399. doi:10.1038/nphoton.2013.57

Yu, Y. H., Jiang, Y., Tang, Z., Guo, Q. L., Jia, J. F., Xue, Q. K., et al. (2005). Thickness dependence of surface plasmon damping and dispersion in ultrathin Ag films. Phys. Rev. B 72, 205405. doi:10.1103/PhysRevB.72.205405

Yuan, Z., and Gao, S. (2008). Landau damping and lifetime oscillation of surface plasmons in metallic thin films studied in a jellium slab model. Surf. Sci. 602, 460-464. doi:10.1016/j.susc.2007.10.040

Yuan, Z., Jiang, Y., Gao, Y., Käll, M., and Gao, S. (2011). Symmetry-dependent screening of surface plasmons in ultrathin supported films: the case of $\mathrm{Al} / \mathrm{Si}(111)$. Phys. Rev. B 83, 165452. doi:10.1103/PhysRevB.83.165452

Zielasek, V., Ronitz, N., Henzler, M., and Pfnür, H. (2006). Crossover between monopole and multipole plasmon of Cs monolayers on $\mathrm{Si}(111)$ individually resolved in energy and momentum. Phys. Rev. Lett. 96, 196801. doi:10.1103/PhysRevLett. 96.196801

Conflict of Interest Statement: The authors declare that the research was conducted in the absence of any commercial or financial relationships that could be construed as a potential conflict of interest.

Received: 22 May 2014; paper pending published: 09 June 2014; accepted: 05 July 2014; published online: 28 July 2014.

Citation: Politano A and Chiarello G (2014) Plasmonic modes in thin films: quo vadis? Front. Mater. 1:9. doi: 10.3389/fmats.2014.00009

This article was submitted to Thin Solid Films, a section of the journal Frontiers in Materials.

Copyright (c) 2014 Politano and Chiarello. This is an open-access article distributed under the terms of the Creative Commons Attribution License (CC BY). The use, distribution or reproduction in other forums is permitted, provided the original author(s) or licensor are credited and that the original publication in this journal is cited, in accordance with accepted academic practice. No use, distribution or reproduction is permitted which does not comply with these terms. 